\title{
A fixed point theorem for cyclic generalized contractions in metric spaces
}

\author{
Maryam A Alghamdi ${ }^{1}$, Adrian Petruşel ${ }^{2}$ and Naseer Shahzad ${ }^{3 *}$
}

${ }^{\text {*Correspondence: }}$

nshahzad@kau.edu.sa

${ }^{3}$ Department of Mathematics, King Abdulaziz University, P.O. Box 80203,

Jeddah, 21859, Saudi Arabia

Full list of author information is

available at the end of the article

\section{Abstract}

In this paper, we extend a recent result of V. Pata (J. Fixed Point Theory Appl. 10:299-305, 2011) in the frame of a cyclic representation of a complete metric space.

\section{Introduction}

One of the fundamental result in fixed point theory is the Banach contraction principle. It has various non-trivial applications in many branches of pure and applied sciences (see, for instance, $[2,7,14]$ and references cited therein).

Let $(X, d)$ be a metric space and $f: X \rightarrow X$ be an operator. We say that $f$ is a contraction if there exists $\lambda \in[0,1)$ such that, for all $x, y \in X$,

$$
d(f(x), f(y)) \leq \lambda d(x, y) .
$$

In terms of Picard operator theory (see [13]), Banach contraction principle asserts that if $f$ is a contraction and $(X, d)$ is complete, then $f$ is a Picard operator. This result has been extended to other important classes of maps. Recently, Pata [8] proved that if $(X, d)$ is a complete metric space and $f: X \rightarrow X$ is an operator such that there exists fixed constants $\gamma \geq 0, \alpha \geq 1$ and $\beta \in[0, \alpha]$ such that, for every $\varepsilon \in[0,1]$ and every $x, y \in X$,

$$
d(f(x), f(y)) \leq(1-\varepsilon) d(x, y)+\gamma \varepsilon^{\alpha} \psi(\varepsilon)[1+\|x\|+\|y\|]^{\beta}
$$

(where $\psi:[0,1] \rightarrow[0, \infty)$ is an increasing function vanishing with continuity at zero and $\|x\|:=d\left(x, x_{0}\right)$, with arbitrary $\left.x_{0} \in X\right)$, then $f$ has a unique fixed point in $X$.

Remark 1.1 (see [8]) The condition (1.2) is weaker than the contraction condition (1.1). In fact, if

$$
d(f(x), f(y)) \leq \lambda d(x, y), \quad \text { for every } x, y \in X \text { and some } \lambda \in[0,1),
$$

then it can be verified that, for every $x, y \in X$, we have

$$
d(f(x), f(y)) \leq(1-\varepsilon) d(x, y)+\gamma \varepsilon^{1+\theta}[1+\|x\|+\|y\|], \quad \text { for every } \theta>0,
$$

(0) 2012 Alghamdi et al.; licensee Springer. This is an Open Access article distributed under the terms of the Creative Commons Attribution License (http://creativecommons.org/licenses/by/2.0), which permits unrestricted use, distribution, and reproduction in any medium, provided the original work is properly cited. 
where

$$
\gamma=\gamma(\theta, \lambda)=\frac{\theta^{\theta}}{(1+\theta)^{1+\theta}} \frac{1}{(1-\lambda)^{\theta}} .
$$

Remark 1.2 (see [8]) The function $f:[1, \infty) \rightarrow[1, \infty)$ defined as

$$
f(x)=-2+x-2 \sqrt{x}+4 \sqrt[4]{x}
$$

has a unique fixed point $x^{*}=1$, but fails to be a contraction on any neighborhood both of 1 and of $\infty$.

Kirk, Srinivasan and Veeramani [6] obtained an extension of Banach's fixed point theorem for mappings satisfying cyclical contractive conditions. Some generalizations of the results given in [6], using the setting of so-called fixed point structures, are presented in I. A. Rus [12]. In [10], Păcurar and Rus established a fixed point theorem for cyclic $\varphi$ contractions and they further discussed fixed point theory in metric spaces. In [3], Karapinar proved a fixed point theorem for cyclic weak $\varphi$-contraction mappings. Some other recent results concerning this topic are given in $[1,4,5,9,11]$.

In the present paper, we obtain a fixed point theorem for a generalized contraction in the sense of the assumption (1.2), defined on a cyclic representation of a complete metric space.

\section{Main results}

We need first to recall a known concept.

Definition 2.1 ([3]) Let $X$ be a nonempty set, $m$ be a positive integer and $f: X \rightarrow X$ an operator. Then, we say that $\bigcup_{i=1}^{m} A_{i}$ is a cyclic representation of $X$ with respect to $f$ if:

(i) $X=\bigcup_{i=1}^{m} A_{i}$, where $A_{i}$ are nonempty sets for each $i \in\{1, \ldots, m\}$;

(ii) $f\left(A_{1}\right) \subset A_{2}, \ldots, f\left(A_{m-1}\right) \subset A_{m}, f\left(A_{m}\right) \subset A_{1}$.

Let $(X, d)$ be a complete metric space. Selecting an arbitrary $x_{1} \in X$, we denote

$$
\|x\|:=d\left(x, x_{1}\right), \quad \text { for all } x \in X .
$$

Our main result is as follows.

Theorem 2.2 Let $(X, d)$ be a complete metric space, $m$ be a positive integer, $A_{1}, \ldots, A_{m}$ be closed nonempty subsets of $X, Y:=\bigcup_{i=1}^{m} A_{i}, \psi:[0,1] \rightarrow[0, \infty)$ be an increasing function vanishing with continuity at zero, and $f: Y \rightarrow Y$ be an operator. Assume that:

1. $\bigcup_{i=1}^{m} A_{i}$ is a cyclic representation of $Y$ with respect to $f$;

2. For every $\varepsilon \in[0,1], x \in A_{i}$, and $y \in A_{i+1}\left(i \in\{1, \ldots, m\}\right.$, where $\left.A_{m+1}=A_{1}\right)$, we have

$$
d(f(x), f(y)) \leq(1-\varepsilon) d(x, y)+\gamma \varepsilon^{\alpha} \psi(\varepsilon)[1+\|x\|+\|y\|]^{\beta},
$$

where $\gamma \geq 0, \alpha \geq 1$ and $\beta \in[0, \alpha]$ are fixed constants. 
Then, we have the following conclusions:

(i) $f$ is a Picard operator, i.e., $f$ has a unique fixed point $x^{*} \in \bigcap_{i=1}^{m} A_{i}$ and the Picard iteration sequence $\left\{f^{n}(x)\right\}_{n \in \mathbb{N}}$ converges to $x^{*}$, for any initial point $x \in Y$;

(ii) the following estimates hold:

$$
\begin{aligned}
& d\left(x_{n}, x^{*}\right) \leq\left\|x^{*}\right\|, \quad n \geq 2 ; \\
& d\left(x_{n}, x_{1}\right) \leq 2\left\|x^{*}\right\|, \quad n \geq 2 .
\end{aligned}
$$

Proof (i) For convenience of notation, if $j>m$, define $A_{j}=A_{i}$ where $i=j \bmod m$ and $1 \leq i \leq m$. Let $x_{1} \in A_{1}$. Starting from $x_{1}$, let $\left\{x_{n}\right\}_{n \geq 1}$ be the Picard iteration defined by the sequence

$$
x_{n}=f\left(x_{n-1}\right)=f^{n-1}\left(x_{1}\right), \quad n \geq 2,
$$

and set $c_{n}=\left\|x_{n}\right\|$. Assume $x_{n} \neq x_{n+1}$ for all $n$. By (2.1), we have

$$
d\left(x_{n}, x_{n+1}\right) \leq d\left(x_{n-1}, x_{n}\right) \leq \cdots \leq d\left(x_{1}, x_{2}\right)=c_{2} .
$$

First, we prove that the sequence $\left(c_{n}\right)_{n \in \mathbb{N}^{*}}$ is bounded. By (2.2) we get that

$$
\begin{aligned}
c_{n} & \leq d\left(x_{n}, x_{n+1}\right)+d\left(x_{n+1}, x_{2}\right)+d\left(x_{2}, x_{1}\right) \leq d\left(x_{n+1}, x_{2}\right)+2 c_{2} \\
& =d\left(f\left(x_{n}\right), f\left(x_{1}\right)\right)+2 c_{2} .
\end{aligned}
$$

Since $x_{1} \in A_{1}$ and $x_{n} \in A_{n}$, from (2.1), we obtain that

$$
\begin{aligned}
c_{n} & \leq(1-\varepsilon) d\left(x_{n}, x_{1}\right)+\gamma \varepsilon^{\alpha} \psi(\varepsilon)\left[1+\left\|x_{n}\right\|+\left\|x_{1}\right\|\right]^{\beta}+2 c_{2} \\
& =(1-\varepsilon) c_{n}+\gamma \varepsilon^{\alpha} \psi(\varepsilon)\left[1+c_{n}\right]^{\beta}+2 c_{2} \\
& \leq(1-\varepsilon) c_{n}+a \varepsilon^{\alpha} \psi(\varepsilon) c_{n}^{\alpha}+b,
\end{aligned}
$$

where $c_{1}=\left\|x_{1}\right\|=d\left(x_{1}, x_{1}\right)=0, \beta \leq \alpha$, and for some $a, b>0$. Thus,

$$
\varepsilon c_{n} \leq a \varepsilon^{\alpha} \psi(\varepsilon) c_{n}^{\alpha}+b
$$

If there is a subsequence $\left(c_{n_{k}}\right)_{k \in \mathbb{N}^{*}} \rightarrow \infty$, the choice $\varepsilon=\varepsilon_{k}=\frac{(1+b)}{c_{n_{k}}}$ leads to the contradiction

$$
1 \leq a(1+b)^{\alpha} \psi\left(\varepsilon_{k}\right) \rightarrow 0 .
$$

Therefore, the sequence $\left(c_{n}\right)$ is bounded.

From (2.2) we obtain that the sequence $\left\{d\left(x_{n}, x_{n+1}\right)\right\}$ is nonincreasing and then it is convergent to the real number

$$
\lim _{n \rightarrow \infty} d\left(x_{n}, x_{n+1}\right)=r=\inf \left\{d\left(x_{n-1}, x_{n}\right): n=2,3, \ldots\right\} .
$$


Now we show that $r=0$. Assume that $r>0$. Let $x_{n} \in A_{n}$ and $x_{n+1} \in A_{n+1}$. By (2.1), we have

$$
\begin{aligned}
r & \leq d\left(x_{n}, x_{n+1}\right)=d\left(f\left(x_{n-1}\right), f\left(x_{n}\right)\right) \\
& \leq(1-\varepsilon) d\left(x_{n-1}, x_{n}\right)+\gamma \varepsilon^{\alpha} \psi(\varepsilon)\left[1+\left\|x_{n-1}\right\|+\left\|x_{n}\right\|\right]^{\beta} \\
& \leq(1-\varepsilon) d\left(x_{n-1}, x_{n}\right)+K \varepsilon \psi(\varepsilon),
\end{aligned}
$$

for some $K>0$. Letting $n \rightarrow \infty$, we obtain

$$
r \leq K \psi(\varepsilon), \quad \text { for every } \varepsilon \in[0,1]
$$

which implies $r=0$. This leads to a contradiction, therefore

$$
\lim _{n \rightarrow \infty} d\left(x_{n}, x_{n+1}\right)=0
$$

For $p \geq 1$, suppose there exists $j, 0 \leq j \leq m-1$, such that $(n+p)-n+j=1 \bmod m$, i.e., $p+j=1 \bmod m$. Now, let $p$ be fixed, $j=0$ and let

$$
q_{n}=n^{\alpha} d\left(x_{n}, x_{n+p}\right)
$$

So, we have

$$
q_{n+1}=(n+1)^{\alpha} d\left(x_{n+1}, x_{n+1+p}\right)=(n+1)^{\alpha} d\left(f\left(x_{n}\right), f\left(x_{n+p}\right)\right) .
$$

Since $p=1 \bmod m, x_{n}$ and $x_{n+p}$ lie in different sets $A_{i}$ and $A_{i+1}$, for some $1 \leq i \leq m$. Then by (2.1) we have

$$
q_{n+1}=(n+1)^{\alpha}(1-\varepsilon) d\left(x_{n}, x_{n+p}\right)+C(n+1)^{\alpha} \varepsilon^{\alpha} \psi(\varepsilon),
$$

where $C=\sup \gamma\left(1+2 c_{n}\right)^{\beta}<\infty$. Choosing for each $n$

$$
\varepsilon=1-\left(\frac{n}{n+1}\right)^{\alpha} \leq \frac{\alpha}{n+1}
$$

the relation (2.3) becomes

$$
q_{n+1} \leq n^{\alpha} d\left(x_{n}, x_{n+p}\right)+C \alpha^{\alpha} \psi\left(\frac{\alpha}{n+1}\right)=q_{n}+C \alpha^{\alpha} \psi\left(\frac{\alpha}{n+1}\right)
$$

Since $q_{0}=0$, it follows that

$$
q_{n}=\sum_{k=1}^{n}\left(q_{k}-q_{k-1}\right) \leq \sum_{k=1}^{n} C \alpha^{\alpha} \psi\left(\frac{\alpha}{k}\right)=C \alpha^{\alpha} \sum_{k=1}^{n} \psi\left(\frac{\alpha}{k}\right) .
$$

Consequently,

$$
d\left(x_{n}, x_{n+p}\right) \leq C\left(\frac{\alpha}{n}\right)^{\alpha} \sum_{k=1}^{n} \psi\left(\frac{\alpha}{k}\right)
$$


This shows that $\left\{x_{n}\right\}$ is a Cauchy sequence in the complete metric space $(Y, d)$ and, thus, it is convergent to a point $y \in Y=\bigcup_{i=1}^{m} A_{i}$. The case $j \neq 0$ similar.

On the other hand, the sequence $\left\{x_{n}\right\}$ has an infinite number of terms in each $A_{i}$, for every $i \in\{1, \ldots, m\}$. Since $(Y, d)$ is complete, in each $A_{i}, i \in\{1, \ldots, m\}$ we can construct a subsequence of $\left\{x_{n}\right\}$ which converges to $y$. Since each $A_{i}$ is closed for $i \in\{1, \ldots, m\}$, we get that $y \in \bigcap_{i=1}^{m} A_{i}$. Then $\bigcap_{i=1}^{m} A_{i} \neq \emptyset$ and we can consider the restriction

$$
g:=f_{\bigcap_{i=1}^{m} A_{i}}: \bigcap_{i=1}^{m} A_{i} \rightarrow \bigcap_{i=1}^{m} A_{i},
$$

which satisfies the conditions of Theorem 1 in [8], since $\bigcap_{i=1}^{m} A_{i}$ is also closed and complete. From this result, it follows that $g$ has a unique fixed point, say $x^{*} \in \bigcap_{i=1}^{m} A_{i}$.

We claim now that for any initial value $x \in Y$, we get the same limit point $x^{*} \in \bigcap_{i=1}^{m} A_{i}$. Indeed, for $x \in Y=\bigcup_{i=1}^{m} A_{i}$, by repeating the above process, the corresponding iterative sequence yields that $g$ has a unique fixed point, say $z \in \bigcap_{i=1}^{m} A_{i}$. Since $x^{*}, z \in \bigcap_{i=1}^{m} A_{i}$, we have $x^{*}, z \in A_{i}$ for all $i \in\{1, \ldots, m\}$ and, hence, $d\left(x^{*}, z\right)$ and $d\left(f\left(x^{*}\right), f(z)\right)$ are well defined. We can write (2.1) in the form

$$
d\left(x^{*}, z\right)=d\left(f\left(x^{*}\right), f(z)\right) \leq(1-\varepsilon) d\left(x^{*}, z\right)+K \varepsilon \psi(\varepsilon),
$$

for some $K>0$. Suppose that $\varepsilon=0$. Then we have

$$
d\left(f\left(x^{*}\right), f(z)\right) \leq d\left(x^{*}, z\right) .
$$

If equality occurs, the relation

$$
d\left(x^{*}, z\right) \leq K \psi(\varepsilon)
$$

is valid for every $\varepsilon \in[0,1]$, which implies $d\left(x^{*}, z\right)=0$. Thus, $x^{*}$ is the unique fixed point of $f$ for any initial value $x \in Y$.

To prove that the Picard iteration converges to $x^{*}$, let us consider $x_{1} \in Y=\bigcup_{i=1}^{m} A_{i}$. Then there exists $i_{0} \in\{1, \ldots, m\}$ such that $x_{n} \in A_{i_{0}}$. As $x^{*} \in \bigcap_{i=1}^{m} A_{i}$ it follows that $x^{*} \in A_{i_{0}+1}$ as well. By the continuity of $f$, we obtain

$$
d\left(f^{n-1}\left(x_{1}\right), x^{*}\right)=d\left(f\left(x_{n-1}\right), x^{*}\right)=d\left(x_{n}, x^{*}\right)=\lim _{p \rightarrow \infty} d\left(x_{n}, x_{n+p}\right) \leq C\left(\frac{\alpha}{n}\right)^{\alpha} \sum_{k=1} n \psi\left(\frac{\alpha}{k}\right) .
$$

Letting $n \rightarrow \infty$, it follows that $\left(x_{n}\right) \rightarrow x^{*}$, i.e., the Picard iteration converges to the unique fixed point of $f$ for any initial point $x_{1} \in Y$.

(ii) Since $x^{*}$ is a fixed point and $x^{*} \in \bigcap_{i=1}^{m} A_{i}$, we obtain that

$$
d\left(x_{n}, x^{*}\right)=d\left(f\left(x_{n-1}\right), f\left(x^{*}\right)\right) \leq d\left(x_{n-1}, x^{*}\right) \leq \cdots \leq d\left(x_{1}, x^{*}\right)=\left\|x^{*}\right\| .
$$

By (2.4), it follows that

$$
d\left(x_{n}, x_{1}\right) \leq d\left(x_{n}, x^{*}\right)+d\left(x^{*}, x_{1}\right) \leq\left\|x^{*}\right\|+d\left(x^{*}, x_{1}\right) \leq 2\left\|x^{*}\right\| .
$$

In view of Remark 1.1, we immediately obtain the following corollary. 
Corollary 2.3 (Kirk, Srinivasan, Veeramani [2, Theorem 1.3]) Let $(X, d)$ be a complete metric space, $m$ be a positive integer, $A_{1}, \ldots, A_{m}$ be closed nonempty subsets of $X, Y:=$ $\bigcup_{i=1}^{m} A_{i}$ and $f: Y \rightarrow Y$ be an operator. Assume that:

(i) $\bigcup_{i=1}^{m} A_{i}$ is a cyclic representation of $Y$ with respect to $f$;

(ii) there exists $\lambda \in[0,1)$ such that, for any $x \in A_{i}, y \in A_{i+1}$, where $A_{m+1}=A_{1}$, we have

$$
d(f(x), f(y)) \leq \lambda d(x, y) .
$$

Then $f$ has a unique fixed point $x^{*} \in \bigcap_{i=1}^{m} A_{i}$.

Finally, we will prove a periodic point theorem. For this purpose, notice first that if $f$ satisfies (1.2) with constants $\alpha, \beta, \gamma$ and function $\psi$, and if $\|f(x)\| \leq\|x\|$ for each $x \in X$, then its $m$-iterate $f^{m}$ also satisfies the condition (1.2) with constants $\alpha, \beta, m \gamma$ and function $\psi$. Indeed, let us suppose that $f$ satisfies (1.2) with constants $\alpha, \beta, \gamma$. Then, for every $\varepsilon \in[0,1]$, we have

$$
\begin{aligned}
& d\left(f^{2}(x), f^{2}(y)\right) \\
& \leq(1-\varepsilon) d(f(x), f(y))+\gamma \varepsilon^{\alpha} \psi(\varepsilon)[1+\|f(x)\|+\|f(y)\|]^{\beta} \\
& \leq(1-\varepsilon)\left[(1-\varepsilon) d(x, y)+\gamma \varepsilon^{\alpha} \psi(\varepsilon)(1+\|x\|+\|y\|)^{\beta}\right] \\
&+\gamma \varepsilon^{\alpha} \psi(\varepsilon)[1+\|f(x)\|+\|f(y)\|]^{\beta} \\
& \leq(1-\varepsilon)\left[(1-\varepsilon) d(x, y)+\gamma \varepsilon^{\alpha} \psi(\varepsilon)(1+\|x\|+\|y\|)^{\beta}\right] \\
&+\gamma \varepsilon^{\alpha} \psi(\varepsilon)[1+\|x\|+\|y\|]^{\beta} \\
&=(1-\varepsilon)^{2} d(x, y)+(1-\varepsilon) \gamma \varepsilon^{\alpha} \psi(\varepsilon)(1+\|x\|+\|y\|)^{\beta} \\
&+\gamma \varepsilon^{\alpha} \psi(\varepsilon)[1+\|x\|+\|y\|]^{\beta} \\
&=(1-\varepsilon)^{2} d(x, y)+(2-\varepsilon) \gamma \varepsilon^{\alpha} \psi(\varepsilon)(1+\|x\|+\|y\|)^{\beta} \\
& \leq(1-\varepsilon) d(x, y)+2 \gamma \varepsilon^{\alpha} \psi(\varepsilon)(1+\|x\|+\|y\|)^{\beta} .
\end{aligned}
$$

Thus, we immediately get that, for $m \in \mathbb{N}$ with $m \geq 2$, we have

$$
d\left(f^{m}(x), f^{m}(y)\right) \leq(1-\varepsilon) d(x, y)+m \gamma \varepsilon^{\alpha} \psi(\varepsilon)(1+\|x\|+\|y\|)^{\beta} .
$$

Notice also that if $\bigcup_{i=1}^{m} A_{i}$ is a cyclic representation of $X$ with respect to $f$, then each $A_{i}$ $(i \in\{1,2, \ldots, m\})$ is an invariant set with respect to $f^{m}$. Using these two remarks, we get the following periodic point theorem.

Theorem 2.4 Let $(X, d)$ be a complete metric space, $m$ be a positive integer, $A_{1}, \ldots, A_{m}$ be nonempty subsets of $X, Y:=\bigcup_{i=1}^{m} A_{i}, \psi:[0,1] \rightarrow[0, \infty)$ be an increasing function vanishing with continuity at zero and $f: Y \rightarrow Y$ be an operator such that $\|f(x)\| \leq\|x\|$ for each $x \in Y$. Assume that:

1. $\bigcup_{i=1}^{m} A_{i}$ is a cyclic representation of $Y$ with respect to $f$.

2. There exists $i_{0} \in\{1, \ldots, m\}$ such that $A_{i_{0}}$ is closed. 
3. For every $\varepsilon \in[0,1]$ and each $x, y \in A_{i_{0}}$, we have

$$
d(f(x), f(y)) \leq(1-\varepsilon) d(x, y)+\gamma \varepsilon^{\alpha} \psi(\varepsilon)[1+\|x\|+\|y\|]^{\beta},
$$

where $\gamma \geq 0, \alpha \geq 1$ and $\beta \in[0, \alpha]$ are fixed constants.

Then, $f^{m}$ has a fixed point.

Proof Notice that, by the above considerations, $f^{m}$ is a self mapping on $A_{i_{0}}$ and it satisfies the condition (1.2) with constants $\alpha, \beta, m \gamma$ and function $\psi$. Thus, by Theorem 1 in [8] we get the conclusion.

\section{Competing interests}

The authors declare that they have no competing interests.

\section{Authors' contributions}

All authors contributed equally and significantly in writing this article. All authors read and approved the final manuscript.

\section{Author details}

${ }^{1}$ Department of Mathematics, Sciences Faculty for Girls, King Abdulaziz University, P.O. Box 4087, Jeddah, 21491, Saudi Arabia. ${ }^{2}$ Department of Mathematics, Babeş-Bolyai University, Kogălniceanu Street No. 1, Cluj-Napoca, 400084, Romania. ${ }^{3}$ Department of Mathematics, King Abdulaziz University, P.O. Box 80203, Jeddah, 21859, Saudi Arabia.

Received: 13 March 2012 Accepted: 4 July 2012 Published: 23 July 2012

\section{References}

1. Agarwal, RP, Alghamdi, M, Shahzad, N: Fixed point theory for cyclic generalized contractions in partial metric spaces. Fixed Point Theory Appl. 2012, 40 (2012)

2. de Bakker, JW, de Vink, EP: Denotational models for programming languages: applications of Banach's fixed point theorem. Topol. Appl. 85(1-3), 35-52 (1998)

3. Karapinar, E: Fixed point theory for cyclic weak $\phi$-contraction. Appl. Math. Lett. 24, 822-825 (2011)

4. Karapinar, E, Erhan, IM, Ulus, AY: Fixed point theorem for cyclic maps on partial metric spaces. Appl. Math. Inf. Sci. 6, 239-244 (2012)

5. Karapinar, E, Sadarangani, K: Fixed point theory for cyclic $(\phi-\psi)$-contractions. Fixed Point Theory Appl. 2011, 69 (2011)

6. Kirk, WA, Srinivasan, PS, Veeramani, P: Fixed points for mappings satisfying cyclical contractive conditions. Fixed Point Theory 4, 79-89 (2003)

7. Kunze, HE, La Torre, D: Solving inverse problems for differential equations by the collage method and application to an economic growth model. Int. J. Optim. Theory Methods Appl. 1(1), 26-35 (2009)

8. Pata, V: A fixed point theorem in metric spaces. J. Fixed Point Theory Appl. 10, 299-305 (2011)

9. Păcurar, M: Fixed point theory for cyclic Berinde operators. Fixed Point Theory 12, 419-428 (2011)

10. Păcurar, M, Rus, IA: Fixed point theory for cyclic $\varphi$-contractions. Nonlinear Anal. 72, 1181-1187 (2010)

11. Petruşel, G: Cyclic representations and periodic points. Stud. Univ. Babeş-Bolyai, Math. 51, 107-112 (2005)

12. Rus, IA: Cyclic representations and fixed points. Ann. Tiberiu Popoviciu Semin. 3, 171-178 (2005)

13. Rus, IA: Picard operators and applications. Sci. Math. Jpn. 58, 191-219 (2003)

14. Serov, VS, Schüermann, HW, Svetogorova, E: Application of the Banach fixed-point theorem to the scattering problem at a nonlinear three-layer structure with absorption. Fixed Point Theory Appl. 2010, Article ID 439682 (2010) 